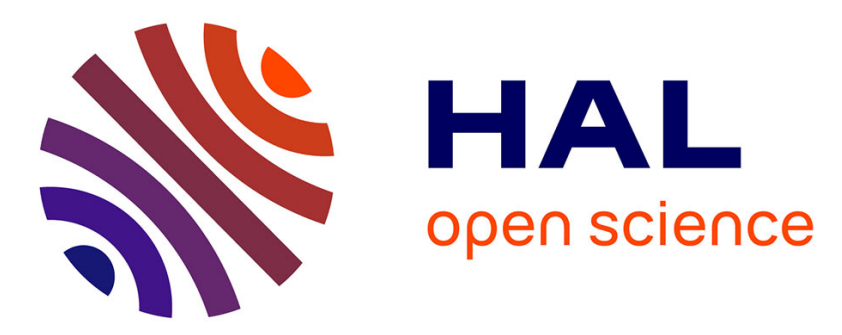

\title{
Levée de la diapause imaginale et reprise de l'activité sexuelle chez la teigne du poireau (Acrolepiopsis assectella) Zell. (Lepidoptera)
}

Ahmed Abo-Ghalia, Eric Thibout

\section{- To cite this version:}

Ahmed Abo-Ghalia, Eric Thibout. Levée de la diapause imaginale et reprise de l'activité sexuelle chez la teigne du poireau (Acrolepiopsis assectella) Zell. (Lepidoptera). Agronomie, 1983, 3 (8), pp.717-722. hal-00884564

\section{HAL Id: hal-00884564 https://hal.science/hal-00884564}

Submitted on 1 Jan 1983

HAL is a multi-disciplinary open access archive for the deposit and dissemination of scientific research documents, whether they are published or not. The documents may come from teaching and research institutions in France or abroad, or from public or private research centers.
L'archive ouverte pluridisciplinaire HAL, est destinée au dépôt et à la diffusion de documents scientifiques de niveau recherche, publiés ou non, émanant des établissements d'enseignement et de recherche français ou étrangers, des laboratoires publics ou privés. 


\title{
Levée de la diapause imaginale et reprise de l'activité sexuelle chez la teigne du poireau (Acrolepiopsis assec- tella) Zell. (Lepidoptera)
}

\author{
Ahmed ABO-GHALIA \& Eric THIBOUT \\ I.B.E.A.S., LA 340 du C.N.R.S., UER Sciences, Université François Rabelais, Parc Grammont, F37200 \\ Tours
}

RÉSUMÉ La levée de la diapause reproductrice chez Acrolepiopsis assectella Zell. ne nécessite aucun facteur particulicr
ct peut être obtenue facilement au laboratoire. Les longues photopériodes et les températures élevées
accélèrent cette levée qui peut atteindre 90 et 100 p. 100 respectivement chez les femelles et les mâles. Chez
ces derniers, la diapause prolonge la période précédant la copulation tandis que, chez les femelles, elle peut
prolonger la période précédant la ponte. L'accroissement de la longévité des individus diapausants par
rapport aux individus mûrs est plus important chez les femelles que chez les mâles.
Dans les conditions expérimentales, après la levée de la diapause, la fécondité des femclles diapausantes cst
inférieure à celle des femelles mûres. De même, la fécondité des femelles diapausantes élevées au laboratoirc
est inférieure à celle des femelles diapausantes récoltées dans la nature en fin de développement larvaire.
D'une façon générale, il semble que plus la période précédant la ponte et la durée de vie sont longues, plus la
fécondité des femelles diapausantes est élevée. Enfin, il semble que la fécondité dépende des conditions
subies avant, pendant et après la diapause. En particulier, des conditions de diapause suffisamment longues et
proches des conditions hivernales permettent une meilleure reprise de l'activité rcproductrice des femelles.

Mots clés additionnels : Diapause reproductrice, longévité, fécondité, photopériode, température.

Termination of the reproductive diapause and resumption of reproductive activity in the leek moth, Acrolepiopsis assectella (Lepidoptera).

The termination of reproductive diapause in the leek moth, Acrolepiopsis assectella, did not need a special
stimulus and could easily be obtained under laboratory conditions. It was accelerated by long photoperiods
and high temperatures and could reach $90 \%$ in the females and $100 \%$ in the males. Reproductive diapause
increased the male pre-copulatory period and sometimes the female pre-oviposition period. The increase in
longevity of diapausing adults compared to mature insects was greater in females than in males.
Under laboratory conditions, the fecundity of mature females was higher than that of diapausing females after
diapause termination. Furthermore, the fecundity of diapausing females reared in the laboratory was lower
than that of diapausing females collected from the field at the end of larval development. The high fecundity
of diapausing females seemed to be correlated with a long pre-oviposition period and a long life. Fecundity
very probably depends on the conditions encountered by the insects before, during and after diapause. Long
and wintery diapausing conditions will particularly favour better resumption of female reproductive activity.

Additional key words : Reproductive diapause, longevity, fecundity, photoperiod, temperature.

\section{INTRODUCTION}

La teigne du poireau, Acrolepiopsis (Acrolepia) assectella Zell. (Hyponomeutoïdea) présente une diapause reproductrice au stade imaginal caractérisée, entre autres, par l'absence d'activité sexuelle chez les 2 sexes, par l'arrêt de la vitellogenèse chez les femelles et de la migration spermatique chez les mâles (THIBOUT, 1981). Cette diapause induite par la photopériode et la température lors du développement larvaire (ABO-GHALIA \& THIBOUT, 1982) permet aux populations septentrionales et du centre de la France de survivre à l'hiver, bien que la mortalité soit cependant élevée (NOYES, 1974). Au printemps, et plus particulièrement vers le début avril dans l'ouest de la France, entre $47^{\circ}$ et $48^{\circ}$ de latitude $\mathrm{N}$ (RAHN, 1970), les teignes reprennent leur activité sexuelle et les $1^{\text {res }}$ pontes peuvent être observées.

L'évolution ultérieure des populations dépendant au moins en partie des effectifs printaniers de la $1^{\text {re }}$ génération, il a paru indispensable de préciser comment se faisait la 
levée de diapause et quelles en étaient les conséquences sur l'activité reproductrice des adultes. En premier lieu, la levée de la diapause a donc été étudiée dans différentes conditions de photopériode et de température. Cette levée de diapause, difficile à dater avec précision comme l'ont souligné TAUBER \& TAUBER (1976), est caractérisée chez les mâles par l'apparition du comportement sexuel suivi de la copulation et chez les femelles par la copulation suivie de la ponte.

D'autre part, les effets de la diapause ont été analysés sur la fécondité, c'est-à-dire sur le nombre d'œufs pondus par les femelles une fois leur diapause terminée.

\section{MATÉRIEL ET MÉTHODES}

Les insectes utilisés sont issus de souches récoltées sur poireau tous les ans, en mai ou juin, à la Bourdaisière $\left(15 \mathrm{~km}\right.$ à l'est de Tours, $47^{\circ} 23$ de latitude $\left.\mathrm{N}\right)$.

Ils sont ensuite élevés au laboratoire sur poireau dans les conditions contrôlées suivantes: photopériode de $18 \mathrm{~h}$ de lumière et $6 \mathrm{~h}$ d'obscurité (L.D. $=18,6$ ), thermopériode de $26^{\circ}-18^{\circ} \mathrm{C}$ et humidité relative de 60 à 80 p. 100 , les 3 facteurs variant de façon synchrone.

Pour obtenir des adultes en diapause reproductrice (D), des œufs pondus dans les conditions précédentes sont placés en conditions inductrices de la diapause (D) : L.D. $=12.12$, $15^{\circ} \mathrm{C}$ constants et 75 p. 100 d'humidité relative environ. Les adultes témoins, sexuellement mûrs sans diapause (M), sont obtenus de la même façon en plaçant des œufs en conditions inductrices de la maturation sexuelle (M): L.D. $=16.8$, $15^{\circ} \mathrm{C}$ constants et 75 p. 100 d'humidité relative (ABO-GHALIA \& THIBOUT, 1982).

En premier lieu, l'influence de la photopériode et de la température sur la levée de la diapause et la fécondité a été étudiée. Après développement larvo-nymphal dans les conditions $M$ et $D$ ci-dessus, les adultes $M$ et $D$, sexés auparavant au stade pré-nymphal, sont transférés le jour de l'émergence dans des conditions de photopériode et de température susceptibles de lever la diapause. Divers lots d'insectes ont été mis à $15^{\circ} \mathrm{C}$ ou à $20^{\circ} \mathrm{C}$ constants en L.D. $=12.12$ ou en L.D. $=16.8$. Chaque mâle était placé avec une femelle vierge $M$ de $1 \mathrm{j}$ en présence d'un morceau de poireau et de coton imbibé d'eau. Les femelles ne pondant pas sans copulation, la date de ponte de la femelle $M$ indique la reprise d'activité sexuelle du mâle et donc la fin de la diapause de celui-ci. Quant aux femelles D, chacune d'elles était placée dans les mêmes conditions avec un mâle $M$ remplacé tous les $5 \mathrm{j}$ par un autre mâle $M$ de $1 \mathrm{j}$. afin d'éviter une baisse de vitalité. La ponte de chaque femelle était relevée quotidiennement jusqu'à sa mort. Chacune d'elles, particulièrement celles n'ayant pas pondu, était disséquée pour vérifier s'il y avait eu copulation.

En outre, l'activité reproductrice des femelles $\mathrm{D}$ élevées au laboratoire a été comparée, à $15^{\circ} \mathrm{C}$ en L.D. $=16.8$, à celle des femelles récoltées au champ sur poireau, en octobre à la fin de leur vie larvaire. Le développement nymphal de ces dernières femelles s'est effectué au laboratoire, en photopériode naturelle et thermopériode non contrôlée ( $15^{\circ}$ à $20^{\circ} \mathrm{C}$ environ).

L'action des conditions d'induction et de levée de la diapause sur la fécondité a été plus précisément étudiée. Deux lots de femelles (I et II) ont été élevés jusqu'à l'émergence en conditions D $\left(15^{\circ} \mathrm{C}, \mathrm{L} . \mathrm{D} .=12.12\right)$ tandis qu'un $3^{\mathrm{e}}$ lot (III) était placé à $15^{\circ} \mathrm{C}$ avec une photopériode dont la photophase décroissait de 15 à $13 \mathrm{~h}$, à raison d'une diminution d'une demi-heure tous les $6 \mathrm{j}$. Cette dernière condition inductrice de la diapause, plus comparable aux conditions photopériodiques naturelles (ABO-GHALIA \& ThibOuT, 1982), pourrait avoir des conséquences différentes de celles des conditions constantes habituellement utilisées. A l'émergence, les 3 lots sont transférés en obscurité constante à $7{ }^{\circ} \mathrm{C}$. Chaque lot est alors divisé en 5 groupes de 20 femelles minimum qui séjournent 20,30 , 40,50 ou $60 \mathrm{j}$ dans ces conditions, avant d'être transférés à leur tour dans 2 conditions permettant la levée de la diapause (aucun développement ovarien n'est observé chez les femclles à $7^{\circ} \mathrm{C}$ en obscurité, même après plus de $80 \mathrm{j}$ ). Ainsi, les 5 groupes d'un des 2 premiers lots de femelles (I) sont placés à $20^{\circ} \mathrm{C}$ en L.D. $=16.8$, tandis que les groupes des lots II et III sont placés à $20^{\circ} \mathrm{C}$ en L.D. $=12.12$. La ponte de chaque femelle en présence d'un morceau de poireau et d'un mâle $M$ est alors suivie quotidiennement jusqu'à sa mort.

\section{RÉSULTATS}

\section{A. Action de la photopériode et de la température sur la levée de la diapause}

Sachant que les femelles $M$ vierges ne pondent pratiquement pas et que les femelles $M$ accouplées pondent de façon importante dès le lendemain de la copulation (THIBOUT, 1974), la date de la $1^{\text {re }}$ ponte de chaque femelle placée avec un mâle $\mathrm{D}$ est un bon indicateur de la date de copulation et donc de la reprise de l'activité sexuelle (levée de la diapause) chez les mâles $\mathrm{D}$. La longévité des mâles $\mathbf{M}$ ainsi que la durée de la période séparant leur émergence du jour précédant la $1^{\mathrm{re}}$ ponte de leur femelle, ou période précédant la copulation, sont données dans le tableau 1. Quelles que soient les conditions d'expérience, les résultats obtenus avec les mâles $\mathrm{M}$ ne sont pas différents et ont donc été regroupés (tabl. 1). Les mâles $M$ ont toujours une durée de vie et une période précédant leur copulation plus courtes que les mâles D.

Chez les mâles $\mathrm{D}$, la température la plus élevée $\left(20^{\circ} \mathrm{C}\right)$ diminue la longévité et surtout la période avant la copulation.

L'action de la photopériode s'exprime plus nettement à $15^{\circ}$ qu'à $20^{\circ} \mathrm{C}$ (tabl. 1). Ainsi, la photophase courte $(12 \mathrm{~h}$ ) allonge la période précédant la copulation et la durée de vie.

Chez les femelles $\mathrm{D}$, les actions de diverses températures et photopériodes ont été analysées sur la fécondité, la longévité et la durée de la période précédant la ponte. Ce dernier critère, impliquant la copulation est un indice de la date de la reprise de l'activité reproductrice et donc de la date de la levée de la diapause des femelles D. Les résultats sont regroupés dans le tableau 2.

Les femelles $\mathrm{M}$ témoins ne présentant pas de différences significatives en fonction de la photopériode à laquelle elles sont exposées, pour une température donnée, leurs performances ont été regroupées. Ces femelles pondent tôt, tandis que les femelles $\mathrm{D}$ doivent attendre au moins une douzaine de jours. De même, la longévité moyenne des femelles $M$ est moindre que celle des divers lots de femelles $D$ dont certaines peuvent vivre jusqu'à $40 \mathrm{j}$. Enfin, la fécondité des femelles $M$ est supérieure à celle des femelles $D$. La comparaison des femelles D entre elles (tabl. 2) montre que, pour une même photopériode, la longévité est plus brève à $20^{\circ} \mathrm{C}$ qu'à $15^{\circ} \mathrm{C}$. La photopériode intervient également puisque, pour une même température, la longévité est plus brève en L.D. $=16.8$ qu'en L.D. $=12.12$. Quant à la fécondité des femelles $\mathrm{D}$ (tabl. 2), elle dépend également des conditions de température et de photopé- 
TABLEAU 1

Longévité et durée (moyenne en j) de la période précédant la copulation de lots de mâles d'A. assectella mûrs (M) et en diapause (D) placés, chacun dès l'émergence, en présence d'une femelle $M$ dans diverses conditions de température et de photopériode. Les moyennes sont suivies de l'écart-type de la moyenne. Les données suivies d'une même lettre, pour une même colonne, ne diffêrent pas significativement au seuil de 0,05 (Analyse de variance).

Longevity and duration of the pre-copulatory period (means \pm S.E.M.; in days) of different groups of mature (M) and diapausing (D) A. assectella males, each placed at emergence with a virgin $M$ female in various conditions of temperature and photoperiod. Means followed by the same letters, in the same column, were not significantly different at the $5 \%$ level (analysis of variance).

\begin{tabular}{|c|c|c|c|c|c|}
\hline \multirow[t]{2}{*}{ Type de $\delta$} & \multicolumn{2}{|c|}{$\begin{array}{l}\text { Conditions de vie } \\
\text { imaginale }\end{array}$} & \multirow{2}{*}{$\begin{array}{l}\text { Nombre de } \\
\text { couples }\end{array}$} & \multirow{2}{*}{$\begin{array}{l}\text { Période avant } \\
\text { la copulation }\end{array}$} & \multirow{2}{*}{$\begin{array}{l}\text { Longévité } \\
\text { moyenne }\end{array}$} \\
\hline & Température & Photopériode & & & \\
\hline \multirow{2}{*}{$\mathrm{D}$} & $20^{\circ} \mathrm{C}$ & $\begin{array}{l}12.12 \\
16.8\end{array}$ & $\begin{array}{l}30 \\
28\end{array}$ & $\begin{array}{l}6,7 \pm 0,5^{a} \\
6,2 \pm 0,5^{a}\end{array}$ & $\begin{array}{l}23,4 \pm 0,8^{a} \\
20,0 \pm 1,1^{b}\end{array}$ \\
\hline & $15^{\circ} \mathrm{C}$ & $\begin{array}{l}12.12 \\
16.8\end{array}$ & $\begin{array}{l}30 \\
30\end{array}$ & $\begin{array}{l}15,5 \pm 1,0^{\mathrm{b}} \\
10,1 \pm 0,9^{\mathrm{c}}\end{array}$ & $\begin{array}{l}33,9 \pm 1,4^{c} \\
26,7 \pm 1,7^{d}\end{array}$ \\
\hline M & $15^{\circ} \mathrm{C}$ & $\begin{array}{c}12.12 \\
\text { et } \\
16.8\end{array}$ & 60 & $1,7 \pm 0,2^{d}$ & $24,4 \pm 0,7^{\mathrm{ad}}$ \\
\hline
\end{tabular}

TABLEAU 2

Moyennes de la longévité, de la durée de la période précédant la ponte (en j) et de la fécondité de lots de femelles d'A. assectella mûres (M) et en diapause $(D)$ placées chacune dès l'émergence en présence d'un mâle $M$ à diverses conditions de température et de photopériode. Voir explications complémentaires tableau 1.

Longevity and duration of the pre-oviposition period (means $\pm S . E . M . ;$ in days) and fecundity of groups of mature (M) and diapausing (D) A. assectella females each placed at emergence with a $M$ male in various conditions of temperature and photoperiod. See further explanations in table 1 .

\begin{tabular}{|c|c|c|c|c|c|c|}
\hline \multirow{2}{*}{ Type de $q$} & \multicolumn{2}{|c|}{$\begin{array}{l}\text { Conditions de vie } \\
\text { imaginale }\end{array}$} & \multirow{2}{*}{$\begin{array}{l}\text { Nombre de } \\
\text { couples }\end{array}$} & \multirow{2}{*}{$\begin{array}{l}\text { Période avant } \\
\text { la ponte }\end{array}$} & \multirow{2}{*}{$\begin{array}{l}\text { Longévité } \\
\text { moyenne }\end{array}$} & \multirow{2}{*}{$\begin{array}{l}\text { Fécondité } \\
\text { moyenne }\end{array}$} \\
\hline & Température & Photopériode & & & & \\
\hline $\mathrm{D}$ & $20^{\circ} \mathrm{C}$ & $\begin{array}{l}12.12 \\
16.8\end{array}$ & $\begin{array}{l}20 \\
22\end{array}$ & $\begin{array}{l}19,3 \pm 1,1^{a} \\
11,8 \pm 0,9^{b}\end{array}$ & $\begin{array}{l}33,4 \pm 1,4^{\mathrm{a}} \\
23,7 \pm 0,7^{\mathrm{b}}\end{array}$ & $\begin{array}{l}93,2 \pm 10,3 \mathrm{a} \\
82,1 \pm 3,0^{\mathrm{b}}\end{array}$ \\
\hline M & $20^{\circ} \mathrm{C}$ & $\begin{array}{c}12.12 \\
\text { et } \\
16.8\end{array}$ & 41 & $2,8 \pm 0,4^{c}$ & $14,5 \pm 0,7^{c}$ & $128,2 \pm 6,3^{c}$ \\
\hline $\mathrm{D}$ & $15^{\circ} \mathrm{C}$ & $\begin{array}{l}12.12 \\
16.8\end{array}$ & $\begin{array}{l}20 \\
20\end{array}$ & $\begin{array}{l}20,1 \pm 2,4^{\text {a }} \\
16,7 \pm 1,0^{\text {a }}\end{array}$ & $\begin{array}{l}39,8 \pm 2,9^{d} \\
32,3 \pm 1,1^{a}\end{array}$ & $\begin{array}{r}116,3 \pm 5,7^{\mathrm{c}} \\
96,5 \pm 7,5^{\mathrm{a}}\end{array}$ \\
\hline $\mathbf{M}$ & $15^{\circ} \mathrm{C}$ & $\begin{array}{c}12.12 \\
\text { et } \\
16.8\end{array}$ & 41 & $2,7 \pm 0,2^{c}$ & $19,3 \pm 0,6^{\mathrm{e}}$ & $144,2 \pm 0,8^{d}$ \\
\hline
\end{tabular}

riode. Elle est plus élevée à $15^{\circ} \mathrm{C}$ qu'à $20^{\circ} \mathrm{C}$ et en L.D. $=12.12$ qu'en L.D. = 16.8. L'évolution quotidienne de la ponte montre le retard important des femelles D (fig. 1). D'autre part, alors que les femelles $M$ présentent des pontes très abondantes les 2 ou $3 \mathrm{j}$ qui suivent la copulation, les femelles $\mathrm{D}$ pondent plus régulièrement chaque jour.

$\mathrm{Si}$ on compare maintenant à $15^{\circ} \mathrm{C}$ en L.D. $=16.8$, les femelles $\mathrm{D}$ du laboratoire à celles prélevées en octobre dans la nature (tabl. 3), on remarque que les périodes précédant la ponte ne sont pas différentes tandis que la longévité et la fécondité des femelles $\mathrm{D}$ venant de la nature sont supérieures à celles des femelles $\mathrm{D}$ du laboratoire. Ces résultats sont confirmés par la figure 2 qui montre l'allongement de la période de ponte des femelles $\mathrm{D}$ de la nature.
La période précédant la ponte des femelles D paraît moins sensible aux conditions photopériodiques et thermiques que les autres critères étudiés.

\section{B. Action des conditions de la diapause sur la fécondité des femelles D}

Les 3 lots de femelles D (I, II, III), ayant séjourné des temps variés pendant leur diapause imaginale à $7{ }^{\circ} \mathrm{C}$, voient leur ponte débuter à des dates diverses une fois placés en conditions permettant la levée de la diapause. La date des $1^{\text {res }}$ pontes dépend en partie des conditions de développement larvo-nymphal, des conditions de vie imaginale et de la durée du séjour en diapause à $7^{\circ} \mathrm{C}$ en obscurité 

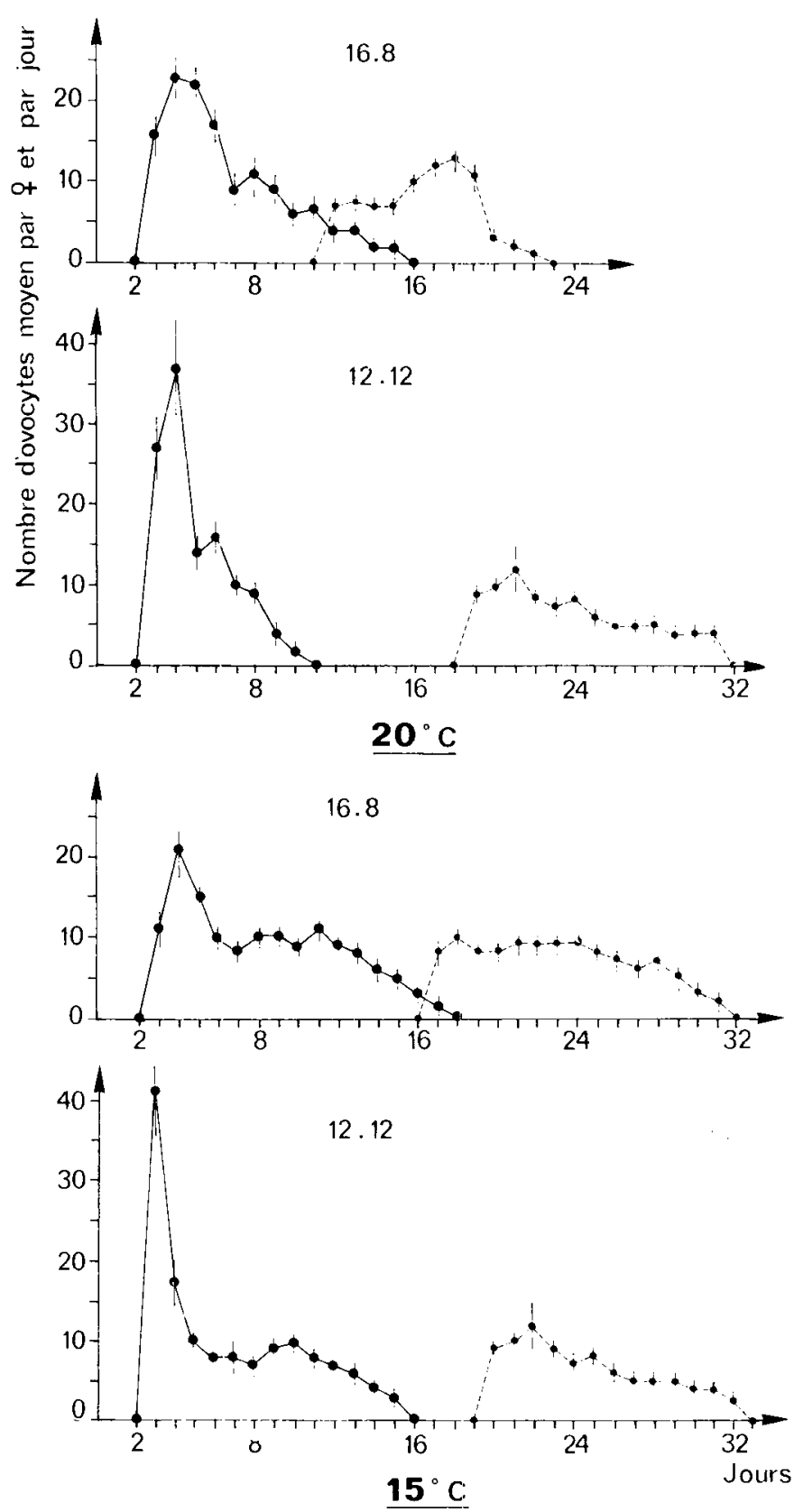

Figure 1

Evolution dans le temps de la ponie quotidienne des femelles d'A. assectclla mûres ( $M$, en trait continu) et en diapause ( $D$, en pointillé) en foncrion des conditions photopériodiques et thermiques après l'émergence.

Daily oviposition of mature females of A. assectella (straight lines) and of diapausing ones (dotted lines) according to their conditions of temperature and photoperiod after emergence.

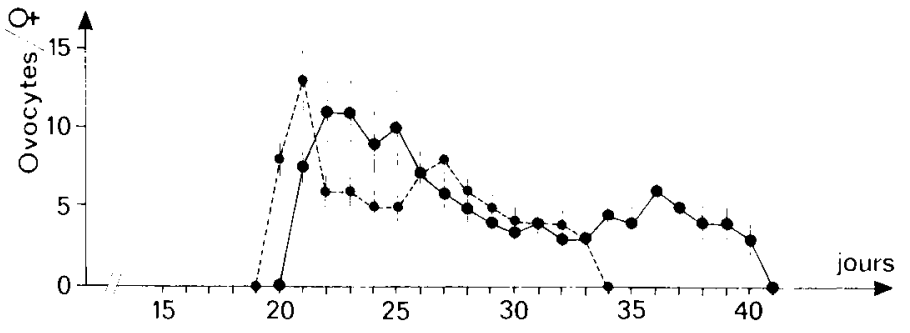

Figure 2

Evolution dans le temps de la ponte quotidienne des femelles $d$ 'A. assectella en diapause prélevées dans la nature ( $N$, en pointillé) ou élevées au laboratoire ( $L$, en trait continu) maintenues après émergence à $15^{\circ} \mathrm{C}$ en L.D. $=16.8$.

Daily oviposition of diapausing females of A. assectella collected in the field (dotted lines) or reared in the laboratory (straight lines) maintained at daylength $=16.8$ at $15^{\circ} \mathrm{C}$ after emergence.

constante (fig. 3). Dans certains cas, la ponte débute d'autant plus vite que la photophase de levée de diapause est plus longue et que la durée du séjour à $7^{\circ} \mathrm{C}$ en obscurité est prolongée.

La fécondité des divers lots de femelles D varie également en fonction des 3 conditions étudiées (tabl. 4). Les pontes les plus importantes sont obtenues chez les femelles élevées aux plus courtes photophases $($ L.D. $=12.12$ ) dont le séjour à $7{ }^{\circ} \mathrm{C}$ en obscurité a été le plus durable $(60 \mathrm{j})$ et qui ont terminé leur diapause reproductrice aux photophases les plus longues (L.D. $=16.8$ ).

Dans cette expérience, il est difficile de déterminer le rôle de la diminution progressive de la photophase (lot III). Cependant, les insectes ont toujours été soumis à des photophases supérieures à $13 \mathrm{~h}$.

\section{DISCUSSION ET CONCLUSION}

Chez $A$. assectella, la levée de la diapause reproductrice, caractérisée ici par la reprise de l'activité sexuelle et par la ponte, est parfaitement possible au laboratoire avec une bonne efficacité puisque, comme pour les adultes M, 90 p. 100 des femelles D et jusqu'à 100 p. 100 des mâles D se reproduisent. Cette levée semble donc plus facile chez les mâles que chez les femelles; d'ailleurs, la période précédant la copulation est nettement plus courte que celle précédant la ponte. Il est cependant difficile de préciser les causes pour lesquelles $10 \mathrm{p}, 100$ des femelles $D$ ne se reproduisent pas.

La levée de diapause peut être obtenue même si les adultes $\mathrm{D}$ sont maintenus dans les conditions $\mathrm{D}$, qui appliquées aux larves, sont inductrices de la diapause. Aussi,

\section{TABLEAU 3}

Moyennes suivies de l'écart-type de la longévité, de la durée de la période précédant la ponte (en j) et de la fécondité de femelles d'A. asscctella en diapause $(D)$ prélevées dans la nature ou venant du laboratoire. Chaque femelle est placée dès l'émergence en présence d'un mâle mûr, à $15^{\circ} \mathrm{C}$ en L. D. $=16,8$. Les données suivies d'une même lettre, pour une même colonne, ne diffèrent pas significativement au seuil 0,05 (Test $t)$.

Longevity, duration of the pre-oviposition period (means \pm S.E.M.; in days) and fecundity of diapausing A. assectella females collected in the field (Nature) or reared in the laboratory (Labo.). Each female was kept with a mature male at daylength $=16.8$, at $15^{\circ} \mathrm{C}$ after emergence. Means followed by the same letter, in the same column, were not significantly different at the $5 \%$ level ( $t$ test).

\begin{tabular}{|c|c|c|c|c|}
\hline & Nombre de couples & $\begin{array}{l}\text { Période avant } \\
\text { la ponte }\end{array}$ & $\begin{array}{l}\text { Longévité } \\
\text { moyenne }\end{array}$ & $\begin{array}{l}\text { Fécondité } \\
\text { moyenne }\end{array}$ \\
\hline D Nature & 23 & $20,8 \pm 0,7^{a}$ & $46,1 \pm 2,3^{a}$ & $138,3 \pm 7,5^{a}$ \\
\hline D Labo. & 23 & $20,4 \pm 2,0^{\text {a }}$ & $33,6 \pm 2,0^{\mathrm{b}}$ & $80,7 \pm 7,5^{b}$ \\
\hline
\end{tabular}


comme chez beaucoup d'espèces d'insectes à diapause hivernale (TAUBER et TAUBER, 1976), A. assectella n'a, semble-t-il, pas besoin d'un stimulus déterminé. En particulier, une période de froid n'est pas indispensable contrairement à certaines autres espèces en diapause imaginale

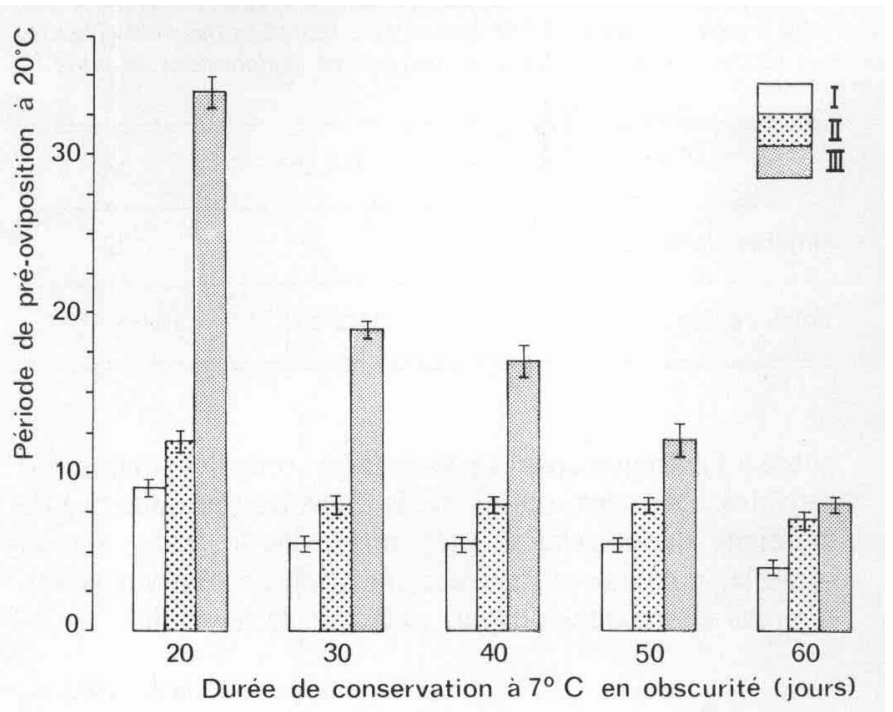

Figure 3

Durée moyenne de la période de préoviposition de 3 lots de femelles $d^{\prime} \mathrm{A}$. assectella en diapause (D), en fonction du temps (en $\left.j\right)$ passé à $7^{\circ} \mathrm{C}$ en $D D$.

I: femelles élevées à $15^{\circ} \mathrm{C}, \quad$ L.D. $=12.12$ puis placées à l'émergence à $20^{\circ} \mathrm{C}$ en L.D. $=16.8$.

II : femelles élevées à $15^{\circ} \mathrm{C}, \quad$ L.D. $=12.12$ puis placées à l'émergence à $20^{\circ} \mathrm{C}$ en L.D. $=12.12$.

III : femelles élevées à $15^{\circ} \mathrm{C}$, avec L.D. diminuant de 15.9 à 13.11 puis placées à l'émergence à $20^{\circ} \mathrm{C}$ en L.D. $=12.12$.

Mean duration of preoviposition period of 3 groups of diapausing A. assectella females according to their time at $7^{\circ} \mathrm{C}$ in continuous darkness.

I: females reared at $15^{\circ} \mathrm{C}$ in daylength: 12.12 and placed at emergence in daylength: 18.6 at $20^{\circ} \mathrm{C}$.

II : females reared at $15^{\circ} \mathrm{C}$ in daylength: 12.12 and placed at emergence in daylength: 12.12 at $20^{\circ} \mathrm{C}$.

III : females reared at $15^{\circ} \mathrm{C}$ with a decreasing photoperiod (from 15.9 to 13.11) and placed at emergence in daylength : 12.12 at $20^{\circ} \mathrm{C}$.
(HODEK, 1974). L'alimentation en eau nécessaire à la levée de la diapause larvaire chez Ostrinia nubilalis (BECK, 1967) peut contribuer à la rapide reprise de l'activité sexuelle de la teigne du poireau en laboratoire. En effet, les basses températures hivernales inhibent pendant une longue période toute activité des adultes $D$ en nature, tandis qu'au laboratoire, les températures utilisées n'ont pas de telles conséquences et les animaux peuvent s'alimenter en eau.

Malgré la rapidité avec laquelle les adultes D reprennent leur activité reproductrice au laboratoire, les périodes précédant la ponte et la copulation, ou souvent la longévité, sont très supérieures à celles des adultes $\mathbf{M}$. Ces périodes vraisemblablement plus longues chez les individus en nature, permettent de synchroniser la période de reproduction avec le retour des conditions favorables du printemps. Inversement, la fécondité des femelles D est inférieure à celle des femelles $M$. Ceci pourrait être la conséquence d'une plus longue consommation d'énergie des femelles D lors de leur diapause et de la période précédant la ponte, la quantité des réserves et donc d'énergie disponible étant déterminée à la fin du développement larvaire.

La comparaison des divers lots de femelles D semble indiquer que leur fécondité dépend à la fois des conditions photopériodiques et thermiques subies lors des stades préimaginaux d'une part, pendant et après la diapause reproductrice lors de la vie imaginale, d'autre part. Ainsi, les femelles $\mathrm{D}$ prélevées à l'extérieur pondent plus que les femelles D du laboratoire où la température est plus élevée et la photophase plus longue qu'en nature à l'automne (tabl. 3). De même, d'après le tableau 4, les femelles ayant la plus faible fécondité sont celles exposées lors de leur développement larvaire à une photophase variable toujours supérieure à $13 \mathrm{~h}$. Il semblerait donc que, chez $A$. assectella, les conditions photopériodiques et thermiques d'induction de la diapause doivent être suffisamment proches des conditions hivernales pour que la fécondité soit élevée.

Si la fécondité est maintenant étudiée en fonction des conditions subies durant la diapause, elle apparaît d'autant plus élevée que la photophase et la température sont plus faibles ou que la durée de la diapause est plus importante (tabl. 2 et 4 ).

\section{TABLEAU 4}

Fécondité moyenne de 3 lots de 20 à 22 femelles d'A. assectella en diapause (D), élevées dans 2 conditions photopériodiques inductrices de la diapause à $15^{\circ} \mathrm{C}$, placées à l'émergence à $7^{\circ} \mathrm{C}$ à l'obscurité constante pendant des temps variant de 20 à 60 j puis transférées chacune en présence d'un mâle mûr, dans 2 conditions photopériodiques à $20^{\circ} \mathrm{C}$. Voir explications complémentaires tableau 1.

Mean fecundity of 3 groups of 20 to 22 diapausing A. assectella females reared at $15^{\circ} \mathrm{C}$ in 2 diapause-inductive photoperiodic conditions, and placed at emergence for various times (from 20 to 60 days) in constant darkness at $7^{\circ} \mathrm{C}$. Each female was then transfered with a mature male to 2 photoperiodic conditions at $20^{\circ} \mathrm{C}$. See further explanation in table 1 .

\begin{tabular}{|c|c|c|c|}
\hline Lots & I & II & III \\
\hline Conditions d'induction de la diapause & \multicolumn{2}{|c|}{$\begin{array}{c}15^{\circ} \mathrm{C} \\
\text { L.D. }=12,12\end{array}$} & $\begin{array}{c}15^{\circ} \mathrm{C} \\
\text { L.D. } 15.9 \text { à } 13.11\end{array}$ \\
\hline Conditions de vie après la diapause & $\begin{array}{c}20^{\circ} \mathrm{C} \\
\text { L.D. }=16.8\end{array}$ & & \\
\hline \multicolumn{4}{|l|}{$\begin{array}{l}\text { Temps à } 7^{\circ} \mathrm{C} \\
\text { en obscurité constante (en j) }\end{array}$} \\
\hline $\begin{array}{l}20 \\
30 \\
40 \\
50 \\
60\end{array}$ & $\begin{array}{l}124,8 \pm 4,2^{\mathrm{c}} \\
136,9 \pm 3,4^{\mathrm{a}} \\
137,1 \pm 3,2^{\mathrm{a}} \\
139,6 \pm 4,2^{\mathrm{ab}} \\
161,4 \pm 11.3^{\mathrm{b}}\end{array}$ & $\begin{array}{c}73,2 \pm 1,5^{\mathrm{d}} \\
119,8 \pm 5,8^{\mathrm{cc}} \\
128,0 \pm 4,9^{\mathrm{ce}} \\
127,8 \pm 4,6^{\mathrm{ac}} \\
136,6 \pm 9,5^{\mathrm{ac}}\end{array}$ & $\begin{array}{r}67,0 \pm 2,8^{\mathrm{g}} \\
91,1 \pm 1,6^{\mathrm{f}} \\
112,3 \pm 4,3^{\mathrm{e}} \\
113,3 \pm 4,1^{\mathrm{e}} \\
103,9 \pm 6,3^{\mathrm{e}}\end{array}$ \\
\hline
\end{tabular}


Enfin, la fécondité peut également être étudiée en fonction des conditions de levée de diapause. Dans ce cas, contrairement aux 2 cas précédents, la fécondité est plus élevée lorsque la photophase est plus longue (tabl. 4).

Une succession de mauvaises conditions de développement et de survie, une profonde et longue diapause, puis de meilleures conditions après la diapause sembleraient accrồtre la fécondité des femelles D. Les effets positifs d'une telle succession, rappelant les conditions naturelles, ont déjà été observés sur la levée de la diapause larvaire chez Ephestia - ellutella Hb. (BELL, 1976). Dans le même ordre d'idée, chez Neodiprion sertifer Geoffr., la morphogenèse qui suit la diapause larvaire est stimulée par les basses températures durant cette diapause et par les fortes températures une fois celle-ci terminée (Wallace \& Sullivan, 1963).

Alors que chez Grapholitha funebrana Treits., les photophases courtes inductrices de la diapause larvaire provoquent la diminution de la fécondité des adultes (DESEO, 1973), chez $A$. assectella, les photophases courtes et la diapause prolongée accroissent la fécondité. Ces différences seraient éventuellement dues, d'une part, chez $G$. funebrana, à une perturbation du comportement alimentaire larvaire après la diapause car les photophases courtes sont appliquées avant pendant et après la diapause larvaire, d'autre part, chez $A$. assectella, à une préservation des réserves larvaires pour la vitellogenèse, du fait d'une activité très réduite des adultes $\mathrm{D}$.

Plus les conditions d'induction de la diapause sont proches des conditions hivernales, plus le poids des prénymphes est élevé (tabl. 5) et plus la fécondité potentielle est importante. RAHN, en 1970, notait que les adultes émer-

\section{TABLEAU 5}

Poids moyen en mg de prénymphes femelles d'A. assectella mûres (M) et en diapause $(D)$ dont le développement larvaire s'est fait en nature ou en laboratoire. Voir explications complémentaires tableau 1.

Mean weight (in $\mathrm{mg}$ ) of mature $(M)$ and diapausing (D) A. assectella female prepupae. Their larvae were reared in the field (Nature) or in the laboratory (Labo.). See further explanations in table 1.

\begin{tabular}{lccc}
\hline \hline & D Nature & D Labo. & M \\
\hline Nombre de $\subsetneq$ & 51 & 32 & 32 \\
\hline Poids en mg & $13,1 \pm 0,6^{\text {a }}$ & $11,2 \pm 0,3^{\mathrm{b}}$ & $10,0 \pm 0,3^{\mathrm{c}}$ \\
\hline \hline
\end{tabular}

geant à l'automne étaient plus gros que ceux des générations estivales. Dans ces conditions, la diapause reproductrice de la teigne du poireau se préparerait dès le stade larvaire sensible, notamment par l'accumulation de réserves larvaires plus abondantes pouvant accroître la fécondité.

Reçu le 27 juillet 1982 Accepté le 29 mars 1983.

\section{REMERCIEMENTS}

Les auteurs remercient le Professeur Vincent LabeYRIE pour ses suggestions et ses critiques. Ils remercient également Messicurs Guy BouRdaIs et Jean-Claude LANDRE pour leur aide technique.

\section{RÉFÉRENCES BIBLIOGRAPHIQUES}

Abo-Ghalia A. H., Thibout E., 1982. Fréquence de la diapause reproductrice en fonction de l'évolution de la photopériode à températures constantes et recherche du stade sensible chez une souche d'Acrolepiopsis assectella (Lepidoptera, Hyponomeutoidea). Ann. Soc. entomol. Fr., 18, 173-179.

Beck S. D., 1967. Water intake and the termination of diapause in the European corn borer, Ostrinia nubilalis. J. Insect Physiol., 13, 739-750.

Bell C. H., 1976. Factors influencing the duration and termination of diapause in the warehouse moth, Ephestia elutella. Physiol. Entomol., 1, 169-178.

Deseo K. V., 1973. Side effect of diapause-inducing factors on the reproductive activity of some lepidopterous species. Nature, 242, 126-127.

Hodek I., 1974. Reactivation of diapausing Aelia acuminata adults before hibernation (Heteroptera). Acta entomol. bohemislov., 71, 65-71.

Noyes J. S., 1974. The biology of the leek moth, Acrolepia assectella (Zeller). Ph. D. Thesis, Univ. London, $254 \mathrm{p}$.
Rahn R., 1970. Rôle de la photopériode sur l'activité de reproduction de la dernière génération d'Acrolepia assectella Zeller (Lepidoptera, Plutellidae) dans l'ouest de la France. C.R. Soc. Biol., 164, 2630-2636.

Tauber M. J., Tauber C. A., 1976. Insect seasonality: diapause maintenance, termination and postdiapause development. Ann. Rev. Entomol., 21, 81-107.

Thibout E., 1974. Influences respectives de la plante-hôte et de la copulation sur la longévité, la ponte, la production ovarienne et la fertilité des femelles d'Acrolepia assectella Zeller (Lepidoptera, Plutellidae). Ann. Zool. Ecol. Anim., 6, 81-96.

Thibout E., 1981. Observations préliminaires et caractérisation de la diapause reproductrice chez la teigne du poireau, Acrolepiopsis assectella (Lepidoptera). Acta Oecol. Oecol. Gen., 2, 171-182.

Wallace D. R., Sullivan C. R., 1963. Laboratory and field investigations of the effect of temperature on the development of Neodiprion sertifer (Geoff.) in the cocoon. Can. Entomol., 95, 1051-1066. 